\title{
ARTICLE
}

\section{Radiation protection aspects in the design of the Linac Coherent Light Source II}

\author{
Mario Santana Leitner*, James C. Liu, Stan X. Mao, Ludovic Nicolas, Sayed H. Rokni and Shanjie Xiao \\ SLAC National Accelerator Laboratory, 2575 Sand Hill Road, Menlo Park, CA, 94025, USA
}

\begin{abstract}
Since 2009, the Linac Coherent Light Source at the SLAC National Accelerator Laboratory produces ultra-fast, ultra-bright X-ray pulses with which atoms and molecules can be visualized as they move, hence revealing the mechanics of chemistry and revolutionizing the research in fields ranging from biology to energy sciences. LCLS-II is a sister vicinal facility with new features that will be soon constructed to address the surging demand of FEL beams. In this paper we summarize the radiation protection scheme for LCLS-II and we describe diverse challenges and the adopted solutions. In particular we present the access modes of LCLS-II that allow simultaneous operation with LCLS. Monte Carlo simulations have been used to design beam components like stoppers and to define the thickness of walls. Also, by carefully analyzing the contributors to the residual dose, the shielding of the main dumps has been optimized to meet engineering constraints while allowing access after short cool down.
\end{abstract}

\section{Keywords: FEL; shielding; Monte Carlo; FLUKA; LCLS; heat deposition; activation}

\section{Introduction}

Since 2009, the Linac Coherent Light Source (LCLS) at the SLAC produces ultra-fast and bright X-ray pulses that allow taking stop images of individual atoms and molecules, thereby unlocking secrets in photosynthesis, catalysis or virus behavior.

Victim of its immediate success, LCLS will soon witness the construction of an annex FEL facility: LCLS-II, which will broaden the range of available X-ray energies with the added possibility to tailor the beam polarization and with increased brightness.

Like its precursor, LCLS-II will use one third of the SLAC two-mile Linac to accelerate electron bunches up to $15 \mathrm{GeV}$, in this case from Linac sector 10 (S10) injector to S20. Those will then travel along the LCLS-I Linac section (S20-S30) using one of the old PEP lines, then through the Beam Switch Yard (BSY) into the head house $(\mathrm{HH})$, where a fast kicker will split the beam into the HXR and SXR beam lines (Figure 1). In the Linac to Undulator-II (LTU-II) section, the two LCLS-II beam lines will cross the SLAC research yard (RY) inside a new beam transport hall (BTH2) that will be constructed at a small angle to the south of the exiting LCLS building (BTH). A new tunnel (UH2) will follow to host two parallel undulator strings that will produce the FEL beams. The electron beams will be bent to buried dumps, while the photons will be conducted by mirror reflections through a thick wall and into the new experimental hall (EH2).
LCLS-II entails radiological concerns of different nature, ranging from prompt high-energy neutron and muon radiation, containment of high power electron and high power density Free Electron Laser (FEL), activation and damage of beam components, or environmental impact to air and groundwater. Moreover, in the identification of sources and paths of radiation, complex operation and access modes need to be studied to allow simultaneous operation of LCLS-II with LCLS and FACET accelerators.

This paper introduces the LCLS-II radiation protection layout and describes a few outstanding cases.

\section{Radiation sources, access modes and radiation protection goals}

\subsection{Radiation sources for normal operation}

The main electron dumps at the end of HXR or SXR, as well as a stopper (D2b) and a single beam dump collimator in the BSY (TDKIKb) are designed to take all the beam power at $120 \mathrm{~Hz}(5 \mathrm{~kW})$. Two additional tune-up dumps in the HXR and SXR lines may be inserted at $10 \mathrm{~Hz}(420 \mathrm{~W})$.

Also, up to $0.1 \%$ of the beam $(5 \mathrm{~W})$ could be lost at any point along the beam line, while in the bends towards the dumps $20 \mathrm{~W}$ of beam-halo could interact with the vacuum chamber generating forward-focused high-energy bremsstrahlung jets of $200 \mathrm{~mW}$. 


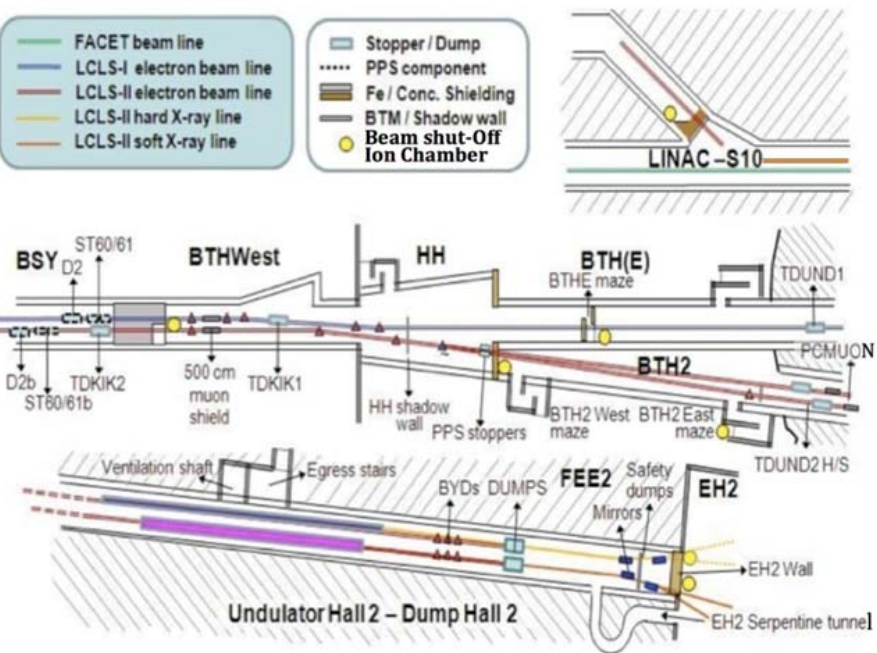

Figure 1. Scheme of LCLS-II beam-line and buildings and of RP-relevant neighboring accelerators.

Certain losses in LCLS-I and in FACET also need to be considered for the design of the shielding walls of LCLS-II, as they may add to the radiation from LCLS-II and/or they may impose access/shielding conditions on some areas of LCLS-II.

Table 1. High-energy radiation sources for LCLS-II shielding design or operation modes. Main sources from neighbor accelerators in italics. Duty factors estimated from LCLS-I operation experience.

\begin{tabular}{rcccc}
\hline$\#$ & $\begin{array}{c}\text { Radiation } \\
\text { Source }\end{array}$ & $\begin{array}{c}\text { Power } \\
(\mathrm{W})\end{array}$ & $\begin{array}{c}\text { Est. duty } \\
\text { Factor }\end{array}$ & Affected areas \\
\hline 1 & FACET-S10 & 10 & medium & S10 inj. Vault \\
2 & D2b & 5000 & $5-15 \%$ & BTHW \\
3 & TDKIKb & 5000 & $1-5 \%$ & BTHW \\
4 & LCLS-TDKIK & 5000 & $1-5 \%$ & RY / BTH2 \\
5 & LCLS-LTU & 5 & unknown & RY / BTH2 \\
6 & LTU2 & 5 & unknown & RY / BTH \\
7 & LCLS-TDUND & 420 & $1-5 \%$ & BTH2 / UH2 \\
8 & TDUND2H/S & 420 & $1-5 \%$ & BTH / UH / hill \\
9 & UH2 & 5 & $<1 \%$ & UH / hill \\
10 & Main bends & 20 & $60-85 \%$ & Hill / EH2 \\
11 & Main dumps & 5000 & $60-85 \%$ & Hill / EH2 \\
\hline
\end{tabular}

\subsection{Regulations and dose limits}

Workers, users, general public and environment on-site and off-site must be protected from the operation of accelerators. A number of regulations and lab-wise guidelines are followed to attain this goal.

As a general rule, areas regularly accessible and occupied by users and general workers should not be exposed to more than $1 \mathrm{mSv}$ per year, which typically translates into hourly dose rates of $0.5 \mu \mathrm{Sv} / \mathrm{h}$ for potentially frequently occupied areas like the EH2 or the open campus to the south of RY. If occupancy is expected to be low, higher dose rates are allowed, i.e. 5 $\mu \mathrm{Sv} / \mathrm{h}$ in RY. Moreover, the roof of the BTH2 has been designed to dose rates of up to $50 \mu \mathrm{Sv} / \mathrm{h}$, taking also into account that beam losses in LTU/LTU2 (\#4 and \#5 in Table 1) should be rare and randomly distributed along the full length of BTH/BTH2. Some other areas, like BTHW and the tunnel above the main dumps, are also designed to $50 \mu \mathrm{Sv} / \mathrm{h}$, but access is controlled and radiological training could be required.

Regulations from the US Department of Energy, Environmental Protection Agency and Regional Water Quality Control Board apply to the radioactivity concentration in air and groundwater. Briefly this means that ${ }^{3} \mathrm{H}$ should remain undetectable, while the 'Maximum Exposed Individual' should not receive more than $100 \mu \mathrm{Sv} / \mathrm{y}$ from activated air.

On top of all these regulatory limits and rules, the exposure ALARA principle should be respected.

\subsection{Access modes}

BTHW and down beam areas will be accessible to General Employee Radiation-Trained (GERT) workers if both LCLS-I and LCLS-II beams don't go past PPS stoppers D2 and D2b, respectively.

In order to grant access to BTH2 through FEE2, it is sufficient that D2b (and its backup stoppers ST60b/61b) are in the beam path, and, if LCLS-I beam goes beyond $\mathrm{D} 2$, then the shutters in HH should also be inserted.

LCLS BTH will not be accessible if LCLS-II beam goes into $\mathrm{HH}$, as that zone would not shielded from beam losses in BTHW or HH.

The Research Yard and the roof of BTH2 shall be accessible regardless of the beam conditions on either machine. Close monitoring will determine whether assumed loss distributions and occupancy factors are adequate, and measures could be deployed accordingly (e.g. limit access, add local shielding).

As for the top of the hill above both undulator tunnels (UH/UH2) and dump areas, and the experimental hall $\mathrm{EH} 2$, these may be occupied by visitors at any time.

\section{Analysis of notable sub-systems}

\subsection{Beam switch yard. Access to BTHW}

In order to access BTHW, LCLS-II beam must be safely terminated at (or before) D2b PPS, and shielding must sufficiently attenuate radiation from that location.

\subsubsection{Energy deposition in D2b}

The D2b PPS stopper assembly is composed of a 1-mm thin titanium alloy spoiler preceding the long D2b copper-tungsten cylinder, which is backed up by two long copper stoppers (ST60b/61b). This system should take up to $5 \mathrm{~kW}$ of high-energy electrons.

FLUKA [1,2] was used to simulate the energy deposition density in D2b and in ST60b/61b for minimum beam size profiles (60 $\mu \mathrm{m}$ RMS) going through the spoiler and into the stoppers.

As shown in Figure 2, FLUKA results were scored in a fine regular cylindrical mesh and also in regions of a size inversely proportional to the heat deposition gradient in those. 


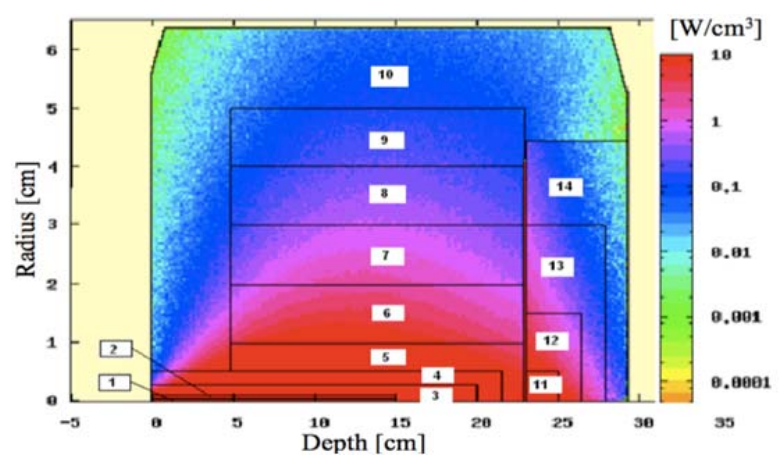

Figure 2. Deposited energy density $\left[\mathrm{W} / \mathrm{cm}^{3}\right]$ in the $\mathrm{D} 2 \mathrm{~b}$ cylinder, for $60 \mu \mathrm{m}$ RMS $15 \mathrm{GeV} \mathrm{e}^{-}$beam on $1 \mathrm{~mm}$ Ti foil $7 \mathrm{~m}$ upstream. The cylinder was divided in 14 zones to score energy deposition for ANSYS evolution analysis.

The latter scoring, less memory-demanding, was used as input for ANSYS 14.0 code, which predicted that, in absence of forced cooling, the steady state temperature would reach $670{ }^{\circ} \mathrm{C}$, while water cooling at $35^{\circ} \mathrm{C}$ would reduce the temperatures to $490{ }^{\circ} \mathrm{C}$ (Figure 3).

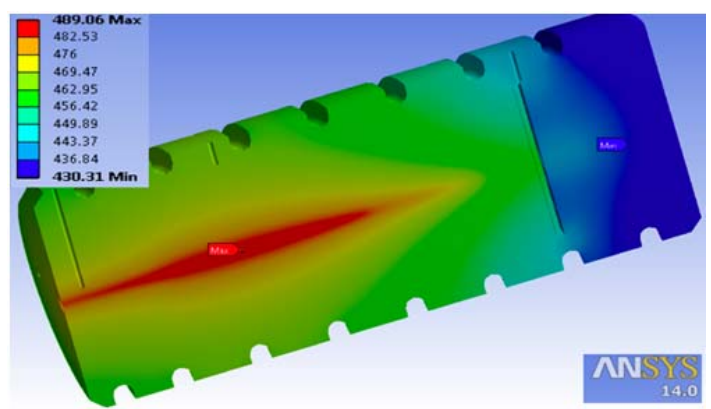

Figure 3. Estimated steady-state temperature [Celsius] of the D2b stopper cooled with $35^{\circ} \mathrm{C}$ water.

\subsubsection{Radiation fields in BTHW}

The Beam Switch Yard BSY-BTHW is a complex and irregular area with three diverging half-tunnels over two floors with some openings for stairs, columns, uneven shielding made of stacked blocks of different size, etc. Initially, a simplified version of this geometry was coded into FLUKA to investigate the adequacy of the thick (12-16 m) iron shielding between BSY and BTHW, including the pipe and the lead casing around it. As expected, dose rates in BTHW were low, with only high-energy muons being sufficiently penetrating to reach the area.

However, when a more realistic 3D model was implemented, simulations showed that neutrons would leak into BTHW through weaknesses in the contact between the BTHW sidewalls or roof and the front iron shielding. As a result, D2b was relocated away from a floor shaft and concrete blocks will cover the seam between shielding elements.

Figure 4 displays a plan view of the muon dose rate and an elevation map for the total dose rate, both at beam planes. The first one demonstrates the inability of radiation to penetrate through the shielding, while the second illustrates how neutrons leak through weak spots.

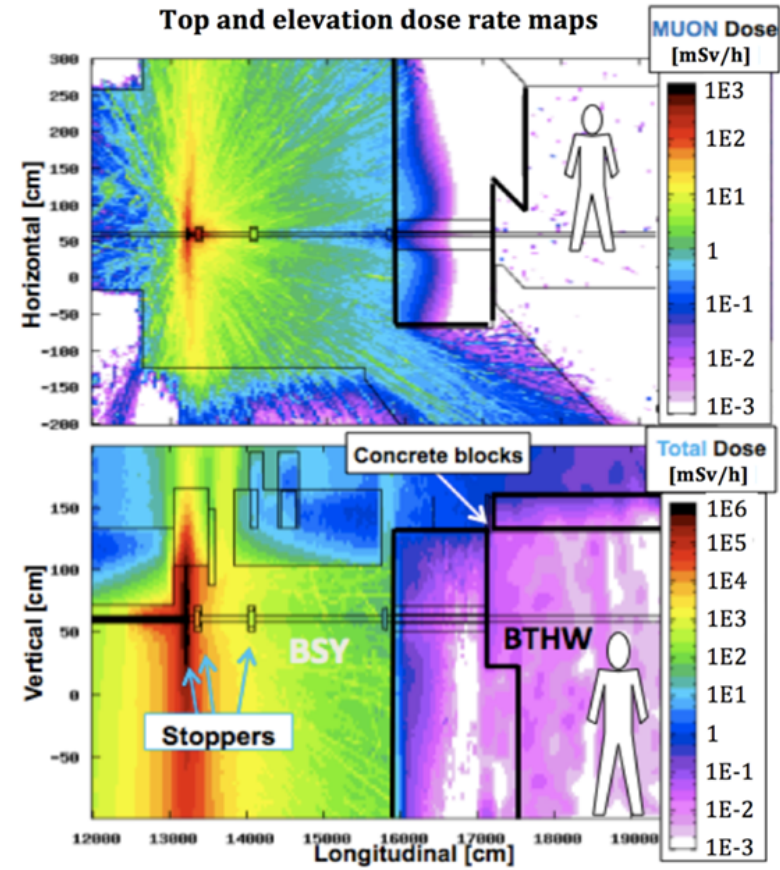

Figure 4. Top view muon dose rate map and elevation total dose rate map for $5 \mathrm{~kW} 15 \mathrm{GeV} \mathrm{e}^{-}$on D2b [3].

Aside for the normal case when all three stoppers of the D2b PPS group are in the beam path, all permutations where two of the three stoppers fail to insert were simulated, and all situations were found to be safe. Those calculations were rapidly obtained by raising the electromagnetic transport energy cutoffs to photomuon production.

\section{2. ВTH2}

The radiological design of BTH (also known as Linac to Undulator, LTU) requires careful consideration (and choice) of both alternative and coincident radiation sources. Examples are given below.

The kicker in HH may split the LCLS-II beam power unevenly between the HXR and SXR lines. Consequently, at a given time, the standard $5 \mathrm{~W}$ LTU2 loss (\#6 in Table 1) could fully occur in either beam line (but only partially in both). For instance, for the design of BTH2 south wall LTU2 losses were assumed exclusively in the SXR, which is the nearest source.

On the other hand, simultaneous $5 \mathrm{~W}$ radiation sources in LTU and LTU2 should be considered for the determination of the roof thickness, as those losses are compatible and the corresponding radiation fields overlap significantly on the roof. In that case, due to its proximity to LCLS-I, it is more conservative to assume that LTU2 losses take place in HXR. Figure 5 illustrates this situation, computed by superimposing the dose rate maps simulated for $5 \mathrm{~W}$ losses in LCLS-I LTU and in LCLS-II SXR. This suggests that a $1.20 \mathrm{~m}$ thick concrete roof is sufficient to limit the dose rate to 50 $\mu \mathrm{Sv} / \mathrm{h}$. 


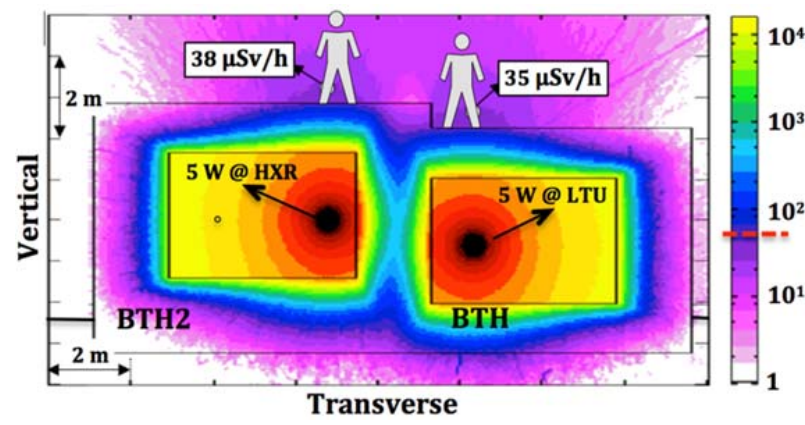

Figure 5. Cross section dose rate map $[\mu \mathrm{Sv} / \mathrm{h}]$ in BTH/BTH2 for simultaneous $5 \mathrm{~W}$ losses in LCLS-I and in LCLS-II SXR.

\subsection{Main beam dumps}

The main electron dumps are buried $90 \mathrm{~cm}$ below the tunnel floor in large cavities filled up mostly by iron, at a location where personnel and equipment transit is expected short after beam off.

The residual dose above the dumps (one month irradiation at $5 \mathrm{~kW}$, one hour cool-down) was brought down from over $500 \mu \mathrm{Sv} / \mathrm{h}$ in the initial design to less than $50 \mu \mathrm{Sv}$ for the optimized shielding, thereby avoiding to break up the tunnel with a Radiation Area. This was achieved through successive simulations, in which the total activation and the contribution from single isotopes were monitored. It was found out that, for the LCLS-II irradiation and access times, residual radiation was dominated by short-lived isotopes (e.g. ${ }^{24} \mathrm{Na},{ }^{15} \mathrm{O},{ }^{28} \mathrm{Al}$ ) in the concrete of the tunnel floor and walls. To suppress that, a $20 \mathrm{~cm}$ concrete shell will surround the dump to contain prompt neutrons within the dump pit, therefore reducing the activation of the tunnel. The dump pit will be covered by a thick iron plate that will shield the high-energy gammas emitted by the activated dump core.

The same model was used to estimate groundwater activation considering 10-year irradiation, $75 \%$ duty factor, $50 \%$ average beam power, $30 \%$ soil humidity, leaching and decay during the slow descent to the ground water level $(\sim 1.3 \mathrm{~m} / \mathrm{y})$. Resulting activities are below detectable, i.e. $250 \mathrm{pCi} / 1$ for ${ }^{3} \mathrm{H}$.

The prompt dose to the upper ground was first simulated for all beam power in one dump. The dose rate histogram was mirrored and added to the original one (then all divided by two) to evaluate the case when beam power is equally distributed. As shown in Figure 6, both scenarios result in equivalent iso-dose curves above the tunnel. The leftmost graph indicates that $5 \mathrm{~m}$ of sandstone are enough to limit the dose from the dumps to $0.5 \mu \mathrm{Sv} / \mathrm{h}$. This is far less than the projected coverage of $12 \mathrm{~m}$. As proven just before, this conclusion is valid for any HXR/SXR power distribution scheme.

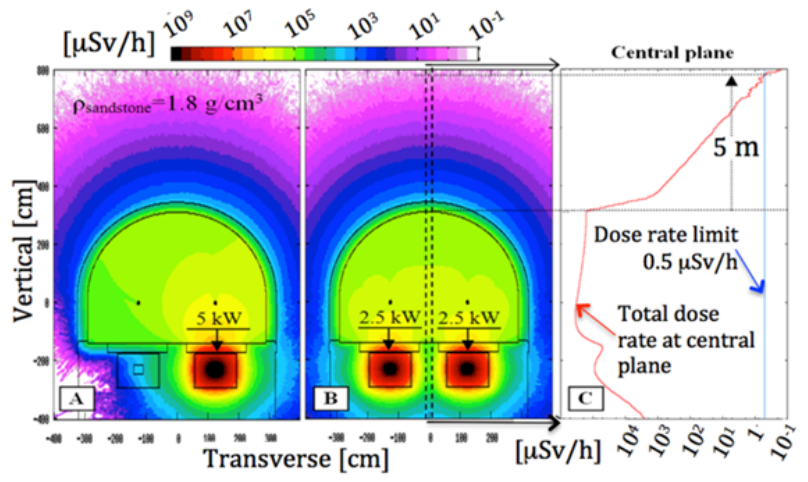

Figure 6. Cross section prompt dose rate maps $[\mu \mathrm{Sv} / \mathrm{h}]$ when A) HXR gets all $5 \mathrm{~kW}, \mathrm{~B}$ ) power is shared between HXR and SXR. C) 1-D projection at tunnel mid plane.

\section{Conclusion}

The radiation protection design of a project like LCLS-II requires careful logic analysis of all beam losses, including those from neighboring machines, followed by agile Monte Carlo simulations.

Further areas of LCLS-II are analyzed in [4].

\section{Acknowledgements}

This work was supported by Department of Energy contract DE-AC02-76-SFO0515.

Steady state temperatures could be obtained thanks to Alev Ibrahimov ANSYS calculations.

\section{References}

[1] G. Battistoni, S. Muraro, P.R. Sala, F. Cerutti, A. Ferrari, S. Roesler, A. Fassò and J. Ranft, The FLUKA code: Description and benchmarking, Proc. of the Had. Shower Sim. Workshop 2006, Fermilab, USA, Sept. 6-8, 2006, M. Albrow and R. Raja eds., AIP Conference Proceed. 896 (2007), pp. 31-49.

[2] A. Ferrari, P.R. Sala, A. Fassò and J. Ranft, FLUKA: A Multi-Particle Transport Code, CERN-2005-10(2005), SLAC-R-773.

[3] V. Vlachoudis, FLAIR: A powerful but user friendly graphical interface, Proc. Int. Conf. on Mathematics, Computational Methods \& Reactor Physics (M\&C 2009), Saratoga Springs, USA, (2009).

[4] S. Xiao, M. Santana-Leitner, L. Nicolas, S. Mao and S. Rokni, Monte Carlo studies for the radiation shielding of LCLS-II, Proc. $12^{\text {th }}$ Int. Conf. on Radiation Shielding (ICRS-12), Nara, Japan, Sep. 2-7, 2012, submitted to Progress in Nucl. \& Technology, (2013). 